\title{
Field \#3 of the Palomar-Groningen Survey
}

\section{Variable stars in the outer edge of the Sagittarius dwarf galaxy}

\author{
Y.K. $\mathrm{Ng}^{1, \star \star}$ and M. Schultheis ${ }^{2}$ \\ 1 IAP, CNRS, 98bis Boulevard Arago, F-75014 Paris, France \\ e-mail: ng@iap.fr \\ ${ }^{2}$ Institut für Astronomie der Universität Wien, Türkenschanzstraße 17, A-1180 Wien, Austria \\ e-mail: schultheis@astro.ast.univie.ac.at
}

Received August 12; accepted September 18, 1996

\begin{abstract}
A catalogue is presented with variable ( $\mathrm{RR}$ Lyrae, semiregular and Mira) stars located inside field \#3 of the Palomar-Groningen Survey at the outer edge of the Sagittarius dwarf galaxy. One of the semiregular variables is a carbon star, comparable with those found by Azzopardi et al. (1991). Serendipity provides the suggestion, that their carbon stars might not be located inside, but behind the bulge in the Sagittarius dwarf galaxy.
\end{abstract}

Key words: stars: carbon; AGB, post AGB; variables: others - local group — galaxies: Sagittarius dwarf

\section{Introduction}

The Palomar-Groningen field \#3 (hereafter referred to as PG3) was searched for variable stars by Plaut (1971). The variables discovered by Plaut were re-examined by Wesselink (1987), using UKST $B_{\mathrm{J}}$ and $R_{\mathrm{F}}$ Schmidt plates. A new blink done on $10 \%$ of the total PG3 area on the original $103 \mathrm{aO}$ plate material used by Plaut, resulted in the discovery of some additional variables. It demonstrated that the completeness of the variable star catalogue is better for variables with larger amplitudes. The variables from this catalogue were the subject of various studies: the RR Lyrae (Oort \& Plaut 1975; Wesselink 1987), the Mira variables (Blommaert 1992), and the semiregular variables (Schultheis et al. 1996, hereafter referred to as Paper II).

Send offprint requests to: Y.K. Ng

* Table 1 and the finding charts of the variable stars are only available in electronic form at the CDS via anonymous ftp to cdsarc.u-strasbg.fr (130.79.128.5) or http://cdsweb.ustrabg.fr/Abstract.html

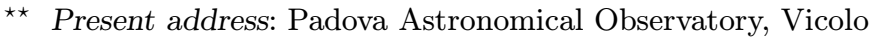
dell'Osservatorio 5, I-35122 Padova, Italy
The non-variable stars in PG3 were studied by Ng (1994) and $\mathrm{Ng}$ et al. (1995).

The Sagittarius dwarf galaxy was first identified by Ibata et al. (1994, hereafter referred to as IGI). Alard (1996) and Mateo et al. (1996) found that this galaxy is spatially more extended than found by IGI. Some stars from the Sagittarius dwarf galaxy could be present in the PG3 variable star catalog from Plaut (1971) and Wesselink (1987).

In this paper we present a small catalogue of PG3 variable stars, which are likely member of the Sagittarius dwarf galaxy. The selection criteria and the catalogue are presented in Sect. 2. A discussion is given in Sect. 3, about the implications, that the carbon star in this sample has, on those found in the direction of the galactic bulge by Azzopardi et al. (1991).

\section{Selection and catalogue}

Alard (1996) demonstrated that the faint peak in a bimodal distribution of the RR Lyrae stars of Bailey type $a b$ is due to a contribution from the Sagittarius dwarf galaxy. Application of this method to the PG3 RR Lyrae stars gives 12 variables of Bailey type $a b$ (hereafter referred to as RRab and $\mathrm{RR} c$ for Bailey type $c$ ) and 4 $\mathrm{RR} c$ stars. Figure 1 shows the distance modulus distribution from which the RR $a b$ stars were selected. Following Wesselink (1987) we adopted for the absolute magnitude of the RRab: $M_{B_{\mathrm{J}}}=0 .{ }^{\mathrm{m}} 79$ and $M_{R_{\mathrm{F}}}=0 .{ }^{\mathrm{m}} 45$. The difference in the distribution between the two passbands is due to extinction. From Fig. 1 we obtain for PG3 a mean colour excess $E\left(B_{\mathrm{J}}-R_{\mathrm{F}}\right)=0.20 \pm 0{ }^{\mathrm{m}} 05$. The stars with distance modulus larger than $17^{\mathrm{m}} 5$ are selected as possible members of the Sagittarius dwarf galaxy. The RRc candidates are selected in a similar way, straight from their magnitude distribution.

This method cannot be applied to select the long 
period variables in the visual passbands, because of their large amplitudes. In the near-infrared passbands the amplitude of their variations is significantly smaller. From the $K$-magnitude distribution (Paper II, Fig. 2) 1 Mira and 5 semiregular variables are thus selected. The actual number of stars might be even larger, because we do not have near-IR photometry for all PG3 long period variables. Table 1 lists the whole catalogue of PG3 variables which are possibly located in the Sagittarius dwarf galaxy. The finding charts $(2 ! 67 \times 2 ! 67$; north is at the top and east is to the left) are only available in electronic form at the CDS. The identification of the stars was made by Wesselink (1987).

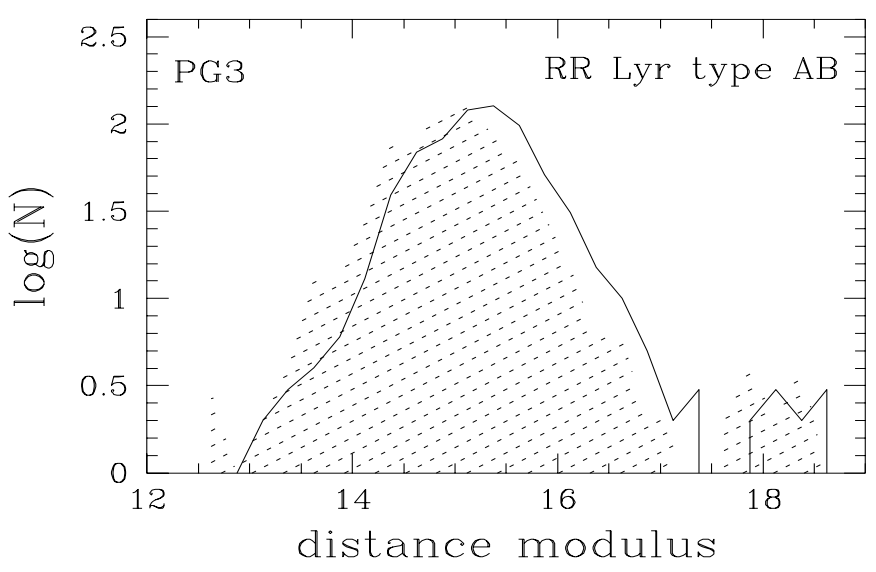

Fig. 1. Distance modulus distribution of the RR Lyrae stars Bailey type $a b$ in PG3 (data from Wesselink 1987, see text for additional details). The solid line shows the distribution obtained from the $B_{\mathrm{J}}$-magnitudes and the dotted distribution is obtained from the $R_{\mathrm{F}}$-magnitudes

\section{Discussion}

The sample is too small to estimate reliably from the RR Lyrae the distance to the Sagittarius dwarf galaxy. Furthermore, we are looking at the edge of the dwarf galaxy and the RR Lyrae sample might be a mixture of stars from the galactic halo and the dwarf galaxy. An indication that we are possibly dealing with a mixed sample can be obtained as follows. Select from the sample those $\mathrm{RR} a b$ stars for which extinction corrections are possible from their colours at minimum light (i.e. $Q=0$ : both the period and the classification are correct). There are only four of those stars in the sample. If the extinction correction is done as described by Wesselink (1987) and Alard (1996) we have to discard two stars from our consideration, because one is too blue (\#1371) and the other one is probably too faint (\#1524). For the two remaining stars we apply the mean reddening correction mentioned in Sect. 2. This gives a mean distance modulus for these stars of $(m-M)_{0}=17^{\mathrm{m}} \cdot 05 \pm 0 .{ }^{\mathrm{m}} 15$ or a distance of $26 \pm 2 \mathrm{kpc}$. The distance modulus is in good agreement with $(m-M)_{0}=17^{\mathrm{m}} \cdot 02 \pm 0 .{ }^{\mathrm{m}} 19$ obtained by Mateo et al. (1995). The distance is within the uncertainties of $24 \pm 2 \mathrm{kpc}$, obtained by Alard (1996) from a much larger sample of stars. The mean distance of the RR $a b$ stars in Table 1 is about $32 \mathrm{kpc}$. This might be due to a contribution of stars from the galactic halo or it might be due to an extension along the line of sight of the dwarf galaxy. Radial velocities of the stars might help to distinguish the two scenarios from each other.

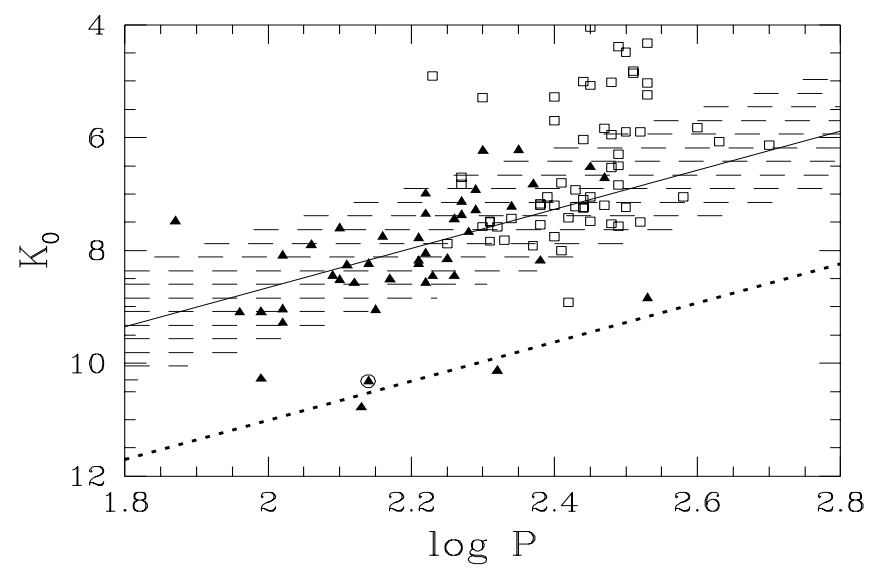

Fig. 2. Period- $K_{0}$ relation for Mira (open square) and semiregular (triangle) variables in PG3. The open circle indicates a carbon star among these variables. The thick solid line is the relation obtained by Glass et al. (1995) for the Miras in Sgr I. The long dashed area shows the contribution in the galactic bulge and the dashed line is the relation from Glass et al., shifted 2.35 (i.e. $26 \mathrm{kpc}$ )

The Mira and semiregular variables in our sample of possible members of the dwarf galaxy are best looked at in a period-luminosity diagram (Fig. 2). Whitelock et al. (1991) demonstrated that the period-luminosity relation is independent of the metallicity of the Miras. This might also apply to the semiregulars. In Paper II it is argued that they are the short period extension to this relation. In Fig. 2 we plotted the variables in Table 1, together with the galactic contribution in PG3. We refer to Paper II for details about the galactic contribution. We also show the $P K_{0}$-relation of Glass et al. (1995) shifted 2m 35 , i.e. to a distance of $26 \mathrm{kpc}$. At this distance the stars are about $4 \mathrm{kpc}$ out of the galactic plane, where one expects to find mainly old metal-poor stars, from the metal-poor thick disc and the halo ( $\mathrm{Ng}$ et al. 1997). In these populations one does not expect to find long period variable stars, because they have not been found in the old metal-poor globular clusters. Therefore, the semiregular variables and Miras cannot be of galactic origin and should belong to a considerable younger population. But a dwarf galaxy, which has interaction with our Galaxy, can contain younger populations. This demonstrates that the long period variables 
could indeed be located in the dwarf galaxy, except for variables \#192 and \#1128 which might have a galactic origin. Additional photometry is required to determine this.

One of the variables (\#283) is a carbon star, see Fig. 3. Details about the spectroscopic observations and the spectral classification of the Miras and the semiregular variables will be given in a forthcoming paper (in preparation). This star appears to be comparable with the carbon stars found in other dwarf galaxies (Aaronson et al. 1983; Azzopardi et al. 1985 \& 1986) and some of the carbon stars found by Azzopardi et al. (1991). Unfortunately, the spectrum of variable \#283 did not include the sodium D doublet and it is not clear if this star is comparable with those found in the "bulge" or the low-metallicity objects in the SMC and the dwarf galaxies. The "bulge" carbon stars have been a mystery (Lequeux 1990; Tyson \& Rich 1991; Westerlund et al. 1991), because they are about $2^{\mathrm{m}} \cdot 5$ in bolometric luminosity too faint to be regarded as genuine AGB stars, if located inside the metal-rich bulge. But if some or all of them are located in the dwarf galaxy, just like variable \#283, there is no need for a metal-rich origin. They are in that case just ordinary metal-poor to intermediate metallicity carbon stars.

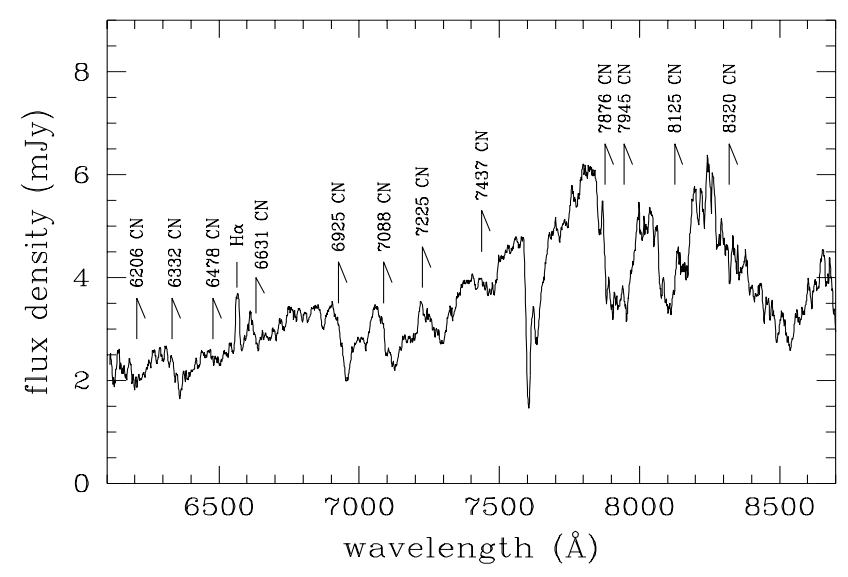

Fig. 3. Medium resolution spectrum smoothed to $2.6 \AA /$ pixel for variable star \#283 (C 3,2), which has a period $P=137.39$ days (Wesselink 1987)

Membership of the dwarf galaxy has important consequences. It implies that the carbon stars are at least younger than approximately 4 Gyr (Marigo et al. 1996 and references cited therein). It indicates that at least 2 major epochs of star formation occurred in the dwarf galaxy, just like the recurrent star formation epochs in the Carina dwarf spheroidal (Schmecker-Hane et al. 1996). It possibly traces the tidal star formation tail due to its passage through our Galaxy. This tail extends at least from $b=-2^{\circ} .4$ to $b=-9.9$, which is far more larger than previously thought. This tail most likely indicates that the Sagittarius dwarf galaxy has passed the galactic plane not so long ago. It would explain the rather blue colours and might also explain the large velocity dispersion of the carbon stars obtained by Tyson \& Rich (1991).

Alksnis (1990) showed that the majority of the carbon stars are SRa-type long period variables. A detailed monitoring of the Azzopardi et al. (1991) carbon stars is required to determine if they are variable and to determine their periods. Together with the period-luminosity relation their membership to the dwarf galaxy can be secured. This can be compared with the properties of the carbon stars studied by Whitelock et al. (1996). Their study indicates the presence of two different groups. One group with $(J-K)_{0}<1$ m 3 is comparable with the carbon stars from Azzopardi et al. (1991), while another group has significantly redder colours $(J-K)_{0}>1$ m. The two groups could be an indication for two different star formation epochs from recent passages through the galactic plane.

AGB stars, like the carbon stars, are the progenitors of planetary nebulae (PN). Along the trail of the Azzopardi et al. (1991) carbon stars one would expect to find long period variables and PNs. The question arises if the PNs found at low galactic latitude with velocities near to that of the Sagittarius dwarf galaxy (Zijlstra \& Walsh 1996) should be considered as true bulge members? Membership of the dwarf galaxy would support the proposition that some or all of the Azzopardi et al. carbon stars do indeed trace the tidal tail.

Acknowledgements. The research of MS is supported by a grant from the Austrian Science Fund under project number P9638AST and S7308. YKN is supported by HCM grant CHRXCT94-0627 from the European Community.

\section{References}

Aaronson M., Olsewski E.W., Hodge P.W., 1983, ApJ 267, 271 Alard C., 1996, ApJ 458, L17

Alksnis A., 1990, in proceedings "From Miras to planetary nebulae: Which path for stellar evolution?", Montpellier (France), Sept. 4-7, 1989, Menessier M.O. and Omont A. (eds.). Editions Frontières, p. 279

Azzopardi M., Lequeux J., Westerlund B.E., 1985, A\&A 144, 388

Azzopardi M., Lequeux J., Westerlund B.E., 1986, A\&A 161, 232

Azzopardi M., Lequeux J., Rebeirot E., Westerlund B.E., 1991, A\&AS 88, 265

Blommaert J.A.D.L., 1992, Ph.D. thesis, Leiden University, the Netherlands

Glass I.S., Whitelock P.A., Catchpole R.M., Feast M.W., 1995, MNRAS 273, 383

Ibata R., Gilmore G., Irwin M.J., 1994, Nat 370, 194

Lequeux J., 1990, in proceedings "From Miras to planetary nebulae: Which path for stellar evolution?", Montpellier (France), Sept. 4-7, 1989, Menessier M.O. and Omont A. (eds.). Editions Frontières, p. 273

Marigo P., Bressan A., Chiosi C., 1996, A\&A 313, 545

Mateo M., Kubiak M., Szymański M., et al., 1995, AJ 110, 1141 
Mateo M., Mirabel N., Udalski A., et al., 1996, ApJ 458, L13 $\mathrm{Ng}$ Y.K., 1994, Ph.D. thesis, Leiden University, the Netherlands

Ng Y.K., Bertelli G., Bressan A., Chiosi C., Lub J., 1995, A\&A 295, 655 (Erratum A\&A 301, 318)

Ng Y.K., Bertelli G., Chiosi C., Bressan A., 1997, A\&A (accepted)

Oort J.H., Plaut L., 1975, A\&A 41, 71

Plaut L., 1971, A\&AS 4, 75

Schmecker-Hane T.A., Stetson P.B., Hesser J.E., VandenBerg D.A., 1996, Proceedings "From Stars to Galaxies". In: Leitherer C., Fritze-von Alvensleben U. and Huchra J. (eds.) ASP Conf. Ser. 98, 328
Schultheis M., Ng Y.K., Hron J., Kerschbaum F., 1996, A\&A (submitted) (Paper II)

Tyson N.D., Rich R.M., 1991, ApJ 367, 547

Wesselink Th.J.H., 1987, Ph.D. thesis, Catholic University Nijmegen, the Netherlands

Westerlund B.E., Lequeux J., Azzopardi M., Rebeirot E., 1991, A\&A 244, 367

Whitelock P.A., Feast M.W., Catchpole R.M., 1991, MNRAS 248, 276

Whitelock P.A., Irwin M., Catchpole R.A., 1996, New Astron. 1,57

Zijlstra A.A., Walsh J.R., 1996, A\&A 312, L21 